\title{
Factor representations of diffeomorphism groups
}

\author{
by \\ Robert P. Boyer (Philadelphia, PA)
}

\begin{abstract}
We give a new construction of semifinite factor representations of the diffeomorphism group of euclidean space. These representations are in canonical correspondence with the finite factor representations of the inductive limit unitary group. Hence, many of these representations are given in terms of quasi-free representations of the canonical commutation and anti-commutation relations. To establish this correspondence requires a generalization of complete positivity as developed in operator algebras. We also compare the asymptotic character formula for the unitary group with the thermodynamic $(N / V)$ limit construction for diffeomorphism group representations.
\end{abstract}

Introduction. The purpose of this paper is to explore the connections between the representation theory of inductive limit classical groups and the representation theory of the group $\operatorname{Diff}_{\mathrm{c}}\left(\mathbb{R}^{d}\right)$ of compactly supported diffeomorphisms of $\mathbb{R}^{d}$. Although the representation theory of this group has been studied for over 25 years because of its many physical applications in quantum mechanics and general relativity, the general representation theory of $\operatorname{Diff}_{\mathrm{c}}\left(\mathbb{R}^{d}\right)$ is still unknown.

The starting point of this work was the discovery that there are natural generalizations of the finite characters of the inductive limit unitary group $U(\infty)$ that yield factor representations of the diffeomorphism group. The importance of this observation is that although $U(\infty)$ is itself too small for most groups to have natural embeddings into it, it does have natural families of representations that do extend to these larger groups in a controlled way.

In Section 1, we investigate the inductive limit of infinite-dimensional unitary groups and study a generalization of positive-definite functions. It is necessary to extend representations from the unitary group to the semigroup of contractions. Much of this parallels the development in $[18,20]$.

In Section 2, we classify a natural class of positive-definite functions which are analogous to the finite characters of $U(\infty)$, called generalized characters. We show that these two collections of positive-definite functions

2000 Mathematics Subject Classification: Primary 22E65; Secondary 46L99, 81R10.

Key words and phrases: diffeomorphism group, factor representation, unitary group. 
are in canonical correspondence. To establish this, it was necessary to use a generalization of complete positivity [1]. The corresponding representations are semifinite factor representations, typically, of type $\mathrm{II}_{\infty}$. Further, these representations may be described in terms of the quasi-free representations of the commutation relations [23]. We do not need to use the classification of finite characters for any of these results.

In Section 3, we apply the results of Sections 1 and 2 to the restrictions of the representations introduced in Section 2 to give new families of factor representations of $\operatorname{Diff}_{\mathrm{c}}\left(\mathbb{R}^{d}\right)$. We also describe the connections between the $N / V$ (thermodynamic) limits $[8,14]$ and the asymptotic character theory of the infinite unitary group $[3,25]$.

In a sequel to this paper, we will introduce further families of generalized characters and study the representations of several natural completions of $\operatorname{Diff}_{\mathrm{c}}\left(\mathbb{R}^{d}\right)$.

I would also like to acknowledge a very helpful conversation with Jerry Goldin.

1. Classical groups and semigroups. It was discovered by Kirillov [12] that certain infinite-dimensional classical groups have countable dual spaces. In particular, the irreducible representations $\pi_{\alpha}$ of the full infinitedimensional orthogonal group are parametrized by a decreasing sequence $\alpha$ of non-negative integers which are eventually zero (called a signature), while the irreducibles of the full unitary group are parametrized by a double signature $(\alpha, \beta)$.

We begin by recording the form of an arbitrary positive-definite function on such groups.

1.1. Proposition. Let $G$ be a separable topological group with only countably many distinct unitary equivalence classes of irreducible representations. Then an arbitrary positive-definite function $f$ on $G$ may be written as

$$
f(g)=\sum_{\pi \in \widehat{G}} \operatorname{Tr}\left[P_{\pi} \pi(g)\right],
$$

where $P_{\pi}$ is a positive trace-class operator on the representation space $H(\pi)$ of $\pi$ such that $\sum_{\pi} \operatorname{Tr}\left[P_{\pi}\right]=1$.

1.2. Definition. Let $L_{1} \subset L_{2} \subset \ldots$ be an increasing sequence of infinite-dimensional separable real Hilbert spaces; let $H_{N}$ be the complexification of $L_{N}$, so we also have a sequence of complex Hilbert spaces. We may form the inductive limit groups:

$$
\mathcal{O}=\underline{\lim } O\left(L_{N}\right), \quad \mathcal{U}=\underline{\lim } U\left(H_{N}\right)
$$

where $O\left(L_{N}\right)$ and $U\left(H_{N}\right)$ are given the strong operator topologies. 
Note. $\mathcal{O}$ is a closed connected subgroup of the topological group $\mathcal{U}$.

We now present a generalization of the result of Ol'shanskil [20, p. 24] that the representation theory of the infinite-dimensional full orthogonal group coincides with the holomorphic representation theory of the full unitary group under restriction. Recall that a representation $\pi$ of the full unitary group is holomorphic if it is a direct sum of irreducible representations $\pi_{\alpha}$ whose signatures $\alpha$ are all non-negative. We say that a representation of $\mathcal{U}$ is holomorphic if its restriction to any $U\left(H_{N}\right)$ is holomorphic.

We include a proof of the following elementary lemma because of its usefulness in this paper.

1.3. Lemma. Let $\left\{\mathcal{M}_{n}\right\}$ and $\left\{\mathcal{N}_{n}\right\}$ be two increasing sequences of ${ }^{*}$-algebras of Hilbert space operators such that (1) $\mathcal{N}_{n} \subset \mathcal{M}_{n}$ for all $n$, and (2) $\left\{\mathcal{M}_{n}\right\}^{\prime \prime}=\left\{\mathcal{N}_{n}\right\}^{\prime \prime}$ for all $n$. Then the following two von Neumann algebras coincide: $\left\{\bigcup \mathcal{M}_{n}\right\}^{\prime \prime}=\left\{\bigcup \mathcal{N}_{n}\right\}^{\prime \prime}$.

Proof. We must show that if $x \in\left\{\bigcup \mathcal{M}_{n}\right\}^{\prime \prime}$, then $x$ lies in the strong closure of $\left\{\bigcup \mathcal{N}_{n}\right\}$. By condition (2), we may assume, without loss of generality, that $x$ does not lie in any $\mathcal{M}_{n}$. So, there must be a sequence $x_{n} \in \mathcal{M}_{n}$ such that $x_{n}$ converges to $x$ in the strong operator topology. Let $\varepsilon>0$ be given together with vectors $\xi_{1}, \ldots, \xi_{k}$. Then there exists a positive integer $N$ such that $n \geq N$ implies $\left\|x_{n} \xi_{j}-x \xi_{j}\right\|<\varepsilon$ for $1 \leq j \leq k$. Again by condition (2), we must have elements $y_{n} \in \mathcal{N}_{n}$ such that $\left\|y_{n} \xi_{j}-x_{n} \xi_{j}\right\|<\varepsilon$ for $1 \leq j \leq k$. Therefore, $\left\|y_{n} \xi_{j}-x \xi_{j}\right\|<2 \varepsilon$ for $1 \leq j \leq k$. We conclude that $x \in\left\{\bigcup \mathcal{N}_{n}\right\}^{\prime \prime}$.

1.4. Corollary. If $\pi$ is a holomorphic unitary representation of $\mathcal{U}$, then the von Neumann algebras generated by $\pi(\mathcal{U})$ and $\pi(\mathcal{O})$ coincide.

Proof. We know that if $\pi_{N}$ is a holomorphic representation of the full unitary group $U\left(H_{N}\right)$, then the von Neumann algebras generated by $U\left(H_{N}\right)$ and its restriction to the orthogonal subgroup $O\left(L_{N}\right)$ agree [20, p. 24]. So, the result follows from the lemma.

1.5. Let $H$ denote the Hilbert space completion of $\bigcup H_{N}$. We choose an orthonormal system $\left\{f_{N}\right\}$ in $H$ such that $f_{1} \in H_{1}$, while $f_{N} \in H_{N} \ominus H_{N-1}$ for $N>1$.

We now form the usual inductive limit unitary group

$$
U(\infty)=\varliminf_{\longrightarrow} U\left(\left\langle f_{1}, \ldots, f_{N}\right\rangle\right) .
$$

If $Y$ is any Hilbert space, we let $\Gamma(Y)$ denote the ${ }^{*}$-semigroup of contractions on $Y$. We note that the unitary group of $Y$ is a ${ }^{*}$-subsemigroup of $\Gamma(Y)$. We next form the inductive limit ${ }^{*}$-semigroup of contractions:

$$
\Gamma(\infty)=\lim _{\longrightarrow} \Gamma\left(\left\langle f_{1}, \ldots, f_{N}\right\rangle\right) .
$$


The following proposition follows easily from the finite-dimensional case.

1.6. Proposition. Any holomorphic unitary representation $\pi$ of $U(\infty)$ extends to $a^{*}$-representation $\widehat{\pi}$ of $\Gamma(\infty)$ with $\{\pi(U(\infty))\}^{\prime \prime}=\{\widehat{\pi}(\Gamma(\infty))\}^{\prime \prime}$.

Proof. Let $\pi$ be given as in the proposition. We consider the restriction $\pi \mid U(N) \simeq \bigoplus_{\alpha} m_{\alpha} \pi_{\alpha}^{(N)}$, where $\pi_{\alpha}^{(N)}$ are irreducible representations of $U(N)$ with multiplicity $m_{\alpha}$. It is classical in finite dimensions that each irreducible representation $\pi_{\alpha}^{(N)}$ extends holomorphically from $U(N)$ to $\Gamma(N)$ so as to preserve multiplication and the adjoint operation. So, $\{\pi(U(N))\}^{\prime \prime}=\{\widehat{\pi}(\Gamma(N))\}^{\prime \prime}$ for all $N$. By Lemma 1.3, we have $\{\pi(U(\infty))\}^{\prime \prime}$ $=\{\widehat{\pi}(\Gamma(\infty))\}^{\prime \prime}$.

1.7. Definition. Let $A$ and $B$ be two topological *-algebras of Hilbert space operators. We call any continuous map (not necessarily linear) $\Psi$ : $A \rightarrow B$ completely positive if, for any $n \geq 1$, the matrix $\left[\Psi\left(a_{i j}\right)\right] \in M_{n}(B)$ is positive for any positive matrix $\left[a_{i j}\right] \in M_{n}(A)$. (This definition is a variant of a definition of Arveson [1].)

If $S_{1}$ and $S_{2}$ are ${ }^{*}$-semigroups such that $S_{1} \subset A$ and $S_{2} \subset B$, where $A$ and $B$ are given above and where these ${ }^{*}$-semigroups are given the relative topologies from $A$ and $B$, then we call a map $\Phi: S_{1} \rightarrow S_{2}$ completely positive if $\Phi$ is the restriction of some completely positive map from $A$ to $B$. We note that if $f: S_{1} \rightarrow \mathbb{C}$ is positive-definite, then $f$ is completely positive.

1.8. Proposition. Any holomorphic positive-definite function of either $U(\infty)$ or $\mathcal{U}$ is completely positive.

Proof. Let $U(\infty)=\underline{\lim } U(N)$, and let $\phi$ be a holomorphic positivedefinite function on $U(\infty)$. To verify that $\phi$ is completely positive, it is enough to verify the condition for the restriction $\phi \mid U(N)$. But this restriction is automatically completely positive by [1]. The argument for $\mathcal{U}$ is similar.

The following corollary follows easily.

1.9. Corollary. The holomorphic extension $\widehat{\phi}$ of a holomorphic positive-definite function $\phi$ of $U(\infty)$ to $\Gamma(\infty)$ is completely positive.

1.10. Comments. Any positive-definite function on a ${ }^{*}$-semigroup $S$ corresponds to a positive linear functional on the discrete ${ }^{*}$-algebra $l^{1}(S)$. In particular, this linear functional is automatically completely positive on this Banach *-algebra. So, the notion of complete positivity for a function on a semigroup or group requires additional structure. We note that not every positive-definite function on the unitary group of a unital $C^{*}$-algebra $A$ is completely positive if $U(A)$ is disconnected (Arveson, unpublished). 


\section{Generalized characters}

2.1. We consider the polarization of $H$ as $H_{+} \oplus H_{-}$, where $H_{-}$is the closed subspace generated by the orthonormal set $\left\{f_{1}, f_{2}, \ldots\right\}$. Let $F$ denote the projection of $H$ onto $H_{-}$, and $F_{N}$ the projection onto $\left\langle f_{1}, \ldots, f_{N}\right\rangle$.

2.2. Proposition. There is a completely positive map $\Phi$ of $\mathcal{U}$ onto $\Gamma(\infty)$ given by compression to the subspace $H_{-}$, that is, $\Phi(W)=F W F$.

Proof. Let $\Phi: B(H) \rightarrow B\left(H_{-}\right)$be given by $\Phi(W)=F W F$. Then $\Phi$ is well known to be completely positive. Now, $\mathcal{U} \subset \bigcup_{N=1}^{\infty} B\left(H_{N}\right)=A \subset B(H)$ and $\Gamma(\infty) \subset B \subset B\left(H_{-}\right)$, where $B$ is the generating nest of $\mathcal{K}+\mathbb{C} \cdot I$ and $A$ and $B$ are given the inductive limit topologies. So, $\Phi(A) \subset B$. Hence, $\Phi: \mathcal{U} \rightarrow \Gamma(\infty)$ is completely positive. Further, any contraction in $B$ is the compression of some unitary element from $A$ by the usual dilation construction. Hence, the map $\Phi$ is onto.

2.3. Corollary. Given a holomorphic positive-definite function $f$ on $U(\infty)$, let $\widehat{f}$ be its unique holomorphic extension to the ${ }^{*}$-semigroup of contractions $\Gamma(\infty)$. Then the composition $\widehat{f} \circ \Phi$ is a positive-definite function on $\mathcal{U}$.

Proof. Let $g_{1}, \ldots, g_{n} \in \mathcal{U}$. Let $A$ and $B$ be the algebras given in the proof of the proposition. Then the matrix $M=\left[g_{j}^{*} g_{i}\right] \in M_{n}(A)$ is positive since $M=M_{0}^{*} M_{0}$, where $M_{0}$ is given with first row $\left[g_{1}, \ldots, g_{n}\right]$ and all other rows zero. Let $f$ be a holomorphic positive-definite function on $U(\infty)$, so it extends to a holomorphic completely positive function $\widehat{f}$ on $\Gamma(\infty)$. Now, the entries of $\left[\Phi\left(g_{j}^{*} g_{i}\right)\right]$ are all in $\Gamma(\infty)$, so we may consider the matrix $\left[\widehat{f}\left(\Phi\left(g_{j}^{*} g_{i}\right)\right)\right]$ of complex numbers, which is positive. Hence, $\widehat{f} \circ \Phi$ is positivedefinite on $\mathcal{U}$.

2.4. We recall that for a pair $(G, L)$, where $G$ is a topological group with closed subgroup $L$, an $L$-central positive-definite function on $G$ is one that is invariant under conjugation by $L$. The set of all such normalized $L$-central positive-definite functions forms a convex set whose extreme points are called generalized characters. For finite or compact groups, $L$-central functions are sometimes called partial traces. Their importance for infinite-dimensional classical groups was noted by Ol'shanskiı̌ [19].

2.5. Lemma. Let $L=\underline{\lim } U(2 \infty)=\underline{\lim } U\left(\left\langle e_{1}, \ldots, e_{N}, f_{1}, \ldots, f_{N}\right\rangle\right)$, with subgroups $K_{1}=U\left(\left\langle e_{1}, e_{2}, \ldots\right\rangle\right) \times I, K_{2}=I \times U\left(\left\langle f_{1}, f_{2}, \ldots\right\rangle\right)$. Then every holomorphic generalized character $\phi$ of the pair $\left(L, K_{2}\right)$ such that $\phi \mid K_{1}=1$ can be written uniquely as $\phi=\hat{\chi} \circ \Phi$, where $\widehat{\chi}$ is the holomorphic extension of a holomorphic finite character $\chi$ from $U(\infty)$ to $\Gamma(\infty)$. 
Proof. Let $\phi$ be a generalized character of $\left(L, K_{2}\right)$ whose restriction to $K_{1}$ is trivial. Set $\phi_{2 n}=\phi \mid U(2 n)$. Then we make the claim that

$$
\phi_{2 n}(\cdot)=\sum_{\alpha} c_{\alpha}^{(2 n)} \operatorname{Tr}\left[\pi_{\alpha}^{(2 n)}\left(E_{n}\right) \pi_{\alpha}^{(2 n)}(\cdot)\right],
$$

where $E_{n}$ is the projection of $\left\langle e_{1}, \ldots, e_{n}, f_{1}, \ldots, f_{n}\right\rangle$ onto $\left\langle f_{1}, \ldots, f_{n}\right\rangle$.

To see this, write $\phi_{2 n}$ as $\sum_{\alpha} \operatorname{Tr}\left[P_{\alpha}^{(2 n)} \pi_{\alpha}^{(2 n)}(\cdot)\right]$, where $P_{\alpha}^{(2 n)}$ is a positive operator on the representation space $H\left(\pi_{\alpha}^{(2 n)}\right)$ and $\sum_{\alpha} \operatorname{Tr}\left[P_{\alpha}^{(2 n)}\right]=1$, by Proposition 1.1. The unitary branching law: $\pi_{\alpha}^{(2 n)} \mid U(n) \times U(n) \simeq \sum_{\beta} \pi_{\beta}^{(n)} \times$ $\pi_{\alpha / \beta}^{(n)}$, together with the condition that $\phi_{2 n} \mid U(n) \times I$ is trivial, implies that the support of the operator $P_{\alpha}^{(2 n)}$ must be contained in the projection onto the subspace where $\pi_{\beta}^{(n)} \times \pi_{\alpha / \beta}^{(n)}$ acts as $\pi_{0}^{(n)} \times \pi_{\alpha}^{(n)}$. In particular, $P_{\alpha}^{(2 n)}=$ $c_{\alpha}^{(2 n)} \pi_{\alpha}^{(2 n)}\left(E_{n}\right)$ for some $c_{\alpha}^{(2 n)} \geq 0$. We further note that the signatures $\alpha$ that contribute to $\phi_{2 n}$ have at most $n$ non-zero entries.

As usual, we let $\pi_{\alpha}^{(2 n)}$ denote the holomorphic extension of $\pi_{\alpha}^{(2 n)}$ from $U(2 n)$ to $\Gamma(2 n)$. Then, by the centrality of the trace, we have

$$
\phi_{2 n}(W)=\sum_{\alpha} c_{\alpha}^{(2 n)} \operatorname{Tr}\left[\widehat{\pi}_{\alpha}^{(2 n)}\left(E_{n} W\right)\right]=\sum_{\alpha} c_{\alpha}^{(2 n)} \operatorname{Tr}\left[\widehat{\pi}_{\alpha}^{(n)}(\Phi(W))\right] .
$$

By (2), we find that $\phi_{2 n}$ is uniquely determined by its restriction to $I \times U(n)$. Moreover, the map $\phi \mapsto \phi \mid I \times U(\infty)$ preserves convex combinations.

Conversely, let $\hat{\chi}$ denote the extension to $\Gamma(\infty)$ of a holomorphic finite character $\chi$ of $U(\infty)$. Then $\hat{\chi} \circ \Phi$ is trivial on $K_{1}$ and conjugate invariant by elements of $K_{2}$. Further, we find that this correspondence is the inverse of the map given above: $\phi \mapsto \phi \mid K_{2}$, since $\widehat{\chi} \circ \Phi \mid K_{2}=\chi$.

REMARK. This argument generalizes the one in [4].

2.6. Lemma. Let $\left\{e_{j}\right\}$ be an orthonormal basis for $H_{+}$such that if $S_{N}=\left\{j: e_{j} \in H_{N}\right\}$, then $\left\{e_{j}: j \in S_{N}\right\} \cup\left\{f_{j}\right\}_{j=1}^{N}$ is an orthonormal basis for $H_{N}$, where $\left\{f_{N}\right\}$ is the orthonormal basis for $H_{-}$as above. Set $U(2 \infty)=\lim _{\longrightarrow} U\left(\left\langle e_{1}, \ldots, e_{N}, f_{1}, \ldots, f_{N}\right\rangle\right)$. Then $U(2 \infty)$ is dense in $\mathcal{U}$.

Proof. We retain the notation of Lemma 2.5. Let $W \in \mathcal{U}$ be given so $W \in$ $U\left(H_{N_{0}}\right)$ for some $N_{0}$. Let $\varepsilon>0$ be given, together with vectors $\xi_{1}, \ldots, \xi_{k}$. Then we need to find $W^{\prime} \in U\left(\left\langle e_{1}, \ldots, e_{n^{\prime}}, f_{1}, \ldots, f_{n^{\prime}}\right\rangle\right)$ for some $n^{\prime}$ such that $\left\|W \xi_{j}-W^{\prime} \xi_{j}\right\|<\varepsilon$ for $1 \leq j \leq k$.

We consider for each $j$ the orthonormal expansion $\xi_{j}=\sum_{n=1}^{\infty} c_{n}^{(j)} e_{n}+$ $\sum_{n=1}^{N_{0}} d_{n}^{(j)} f_{n}$. Choose the index $n^{\prime}$ so that $\left\|\sum_{n=n^{\prime}}^{\infty} c_{n}^{(j)} e_{n}\right\|<\varepsilon / 2$ for $1 \leq j \leq k$. We note that since $\xi_{j} \in H_{N_{0}}$ the coefficients $c_{n}^{(j)}$ are 0 if $e_{n} \notin H_{N_{0}}$. For this reason, we find that $W\left(\sum_{n=1}^{n^{\prime}} c_{n}^{(j)} e_{n}\right) \in H_{N_{0}}$. Hence, there exists $W^{\prime} \in$ 
$U\left(\left\langle e_{1}, \ldots, e_{n^{\prime}}, f_{1}, \ldots, f_{n^{\prime}}\right\rangle\right)$ such that $\left\|W \xi_{j}-W^{\prime} \xi_{j}^{\prime}\right\|<\varepsilon / 2$ for $1 \leq j \leq n$, where $\xi_{j}^{\prime}=\sum_{n=1}^{n^{\prime}} c_{n}^{(j)} e_{n}$. This is the desired $W^{\prime}$, and the lemma is proven.

EXAMPLE. There are non-holomorphic generalized characters of the above pair, satisfying the triviality condition. One such example is

$$
\operatorname{det}((I-F) W) \operatorname{det}\left((I-F) W^{*}\right) .
$$

2.7. Proposition. (a) The convex sets $C_{1}$ and $C_{2}$ are affinely isomorphic, where $C_{1}$ is the set of all holomorphic positive-definite functions on $\mathcal{U}$ that are $I \times U(\infty)$-conjugate invariant and whose restriction to $\left(U\left(H_{+}\right) \cap \mathcal{U}\right) \times I$ is trivial, and where $C_{2}$ is the set of all central holomorphic positive-definite functions on $U(\infty)$.

(b) Let $\phi$ be any holomorphic generalized character from $C_{2}$. Then there exists a unique holomorphic finite character $\chi$ of $U(\infty)$ such that $\phi=\hat{\chi}^{\circ} \Phi$.

Proof. Let $\phi$ be a generalized character of the pair $(\mathcal{U}, I \times U(\infty))$. Then $\phi \mid U(2 \infty)$ is a generalized character as well of $(U(2 \infty), I \times U(\infty))$, for a version of $U(2 \infty)$ that satisfies Lemma 2.6. Further, since $U(2 \infty)$ is dense in $\mathcal{U}, \phi$ is uniquely determined by its restriction to $U(2 \infty)$. By Corollary 2.3, $\phi=\hat{\chi} \circ \Phi$ on $U(2 \infty)$. But $\hat{\chi} \circ \Phi$ is continuous on $U(2 \infty)$ relative to the $\mathcal{U}$-topology. Hence, $\phi=\widehat{\chi} \circ \Phi$ on $\mathcal{U}$ uniquely. This ends the proof.

2.8. Corollary. Let $\chi$ denote a holomorphic finite character of $U(\infty)$. Then $\hat{\chi} \circ \Phi$ is a generalized character of $\mathcal{U}$, which generates either an irreducible representation or a type $\mathrm{II}_{\infty}$ factor representation. In particular, the generalized character is irreducible if and only if its restriction to $U(\infty)$ is a power of the determinant; it is type $\mathrm{II}_{\infty}$ if and only if its restriction to $U(\infty)$ is type $\mathrm{II}_{1}$.

Proof. We make use of the identification [19] of the generalized characters as spherical functions of the pair $(L \times K, K \times K)$. If $f$ is the generalized character with corresponding cyclic representation $T=\pi \times \pi^{\prime}$ of $L \times K$, then $T$ is irreducible, $\pi^{\prime}$ is a finite factor, and $\pi$ and $\pi^{\prime}$ generate each other's commutants. Hence, $\pi$ is irreducible $\Leftrightarrow \pi^{\prime}$ is irreducible. This occurs if and only if $\pi^{\prime}$ is equivalent to a power of the determinant. Otherwise, $\pi^{\prime}$ is always equivalent to a type $\mathrm{II}_{1}$ factor representation. So, $\pi$ must be type II as well. Since $U\left(H_{N}\right)$, so $\mathcal{U}$ itself, has no infinite-dimensional finite factor representations, we find that $\pi$ is type $\mathrm{II}_{\infty} \Leftrightarrow \pi^{\prime}$ is type $\mathrm{II}_{1}$.

2.9. Let $\phi$ be a generalized character given as in 2.7(b): $\phi=\widehat{\chi} \circ \Phi$. Then its restriction has the form

$$
\phi(\cdot) \mid U\left(H_{N}\right)=\sum_{\alpha} a_{\alpha}^{(N)} \operatorname{Tr}\left[\pi_{\alpha}^{(N)}\left(F_{N}\right) \pi_{\alpha}^{(N)}(\cdot)\right],
$$

where $\chi \mid U(N)=\sum_{\alpha} a_{\alpha}^{(N)} \chi_{\alpha}^{(N)}$ (and $\chi_{\alpha}^{(N)}$ is the irreducible character of 
$U(N)$ with signature $\alpha$ and dimension $\left.d_{\alpha}^{(N)}\right)$. In particular, this summation gives us the decomposition of the cyclic representation $\pi_{\phi}$ corresponding to a generalized character:

$$
\pi_{\phi} \mid U\left(H_{N}\right) \simeq \bigoplus_{\alpha} d_{\alpha}^{(N)} \pi_{\alpha}^{(N)} .
$$

Further, we recall from $[2,25,26]$ the explicit form of the holomorphic finite characters of $U(\infty): \chi(W)=\operatorname{det}[f(W)]$, where

$$
f(z)=e^{\gamma(z-1)} \prod_{j=1}^{\infty} \frac{1-\beta_{j}+\beta_{j} z}{1+\alpha_{j}-\alpha_{j} z}
$$

such that $0 \leq \beta_{j+1} \leq \beta_{j} \leq 1,0 \leq \alpha_{j+1} \leq \alpha_{j}$, and $0 \leq \gamma$, together with $\sum_{j=1}^{\infty}\left(\alpha_{j}+\beta_{j}\right)<\infty$. We denote the collection of all such meromorphic functions by $\mathcal{M}$.

We can restate Corollary 2.8 relative to the parametrization of the holomorphic finite characters.

2.10. Corollary. If $\chi(W)=\operatorname{det}[f(W)], f \in \mathcal{M}$, is a finite holomorphic character of $U(\infty)$, then the generalized character $\hat{\chi} \circ \Phi$ is irreducible if and only if $\gamma=0, \alpha_{j}=0$ for all $j$, and there exists an integer $m$ so that $\beta_{j}=1$ for $1 \leq j \leq m$ and $\beta_{j}=0$ for $j>m$, that is, $f(z)=z^{m}$. Otherwise, $\hat{\chi} \circ \Phi$ generates a type $\mathrm{II}_{\infty}$ factor representation.

2.11. Examples. (1) Consider the subgroup $K=U(1)=S^{1} \cdot e_{1}$ of the unitary group $U(N)$. Then every multiplicative character $\chi$ of $K$ admits a unique holomorphic extension to the ${ }^{*}$-semigroup $\Gamma(1)$, which is the unit disk. Note that these representations are all one-dimensional. The compression of a unitary element $W$ to $\Gamma(1)$ is just the matrix coefficient $\left(W e_{1}, e_{1}\right)$. So the above construction amounts to identifying $\chi$ as the matrix coefficient of the standard irreducible representation of $U(N)$ on $\mathbb{C}^{N}$.

(2) Two important examples of finite characters are: $\chi_{+}(W)=\operatorname{det}(1+$ $\alpha-\alpha W)^{-1}$ and $\chi_{-}(W)=\operatorname{det}(1-\beta+\beta W)$, where $\chi_{+}$corresponds to a certain quasi-free state on the Weyl (CCR) algebra and $\chi_{-}$to a certain quasi-free state on the Clifford (CAR) algebra.

Comments. (1) The construction of the positive-definite function on $\mathcal{U}$ as the composition $\hat{\chi} \circ \Phi$ may be viewed as a non-linear version of the Rieffel construction of induced representations of $C^{*}$-algebras. The above construction is not, though, given exactly by induced representations of $C^{*}$ algebras since such induction preserves traceability and this construction does not.

(2) Corollary 2.8 is analogous to results on extremal states relative to an asymptotically abelian sytem on a $C^{*}$-algebra. 
(3) The generalized characters are among the KMS-functions of positive type in [23]. There are no general results concerning their associated representations according to their factoriality, type, or quasi-equivalence.

(4) None of the semifinite factor representations given by the above generalized characters yield traceable factor (normal) representations for $C^{*}(U(2 \infty))$ (see $\left.[2,6]\right)$.

\section{Diffeomorphism group representations}

3.1. Let $X$ denote the euclidean space $\mathbb{R}^{d}$, where $d \geq 2$. Let $Y$ denote an open subset of $X$. We let $\operatorname{Diff}_{\mathrm{c}}(Y)$ denote the group of all compactly supported diffeomorphisms of $Y$ with the usual topology. That is, a diffeomorphism $\psi$ has compact support if it is equal to the identity on the complement of some relatively compact open subset of $X$. Further, a sequence of compactly supported diffeomorphisms $\left\{\psi_{n}\right\}$ converges to $\psi$ if there is a common relatively compact open set on whose complement all diffeomorphisms are equal to the identity and, on the open set itself, the diffeomorphisms converge in the $\mathcal{C}^{\infty}$ topology [24].

Let $\mathcal{F}$ denote an increasing sequence $X_{1} \subset X_{2} \subset \ldots$ of connected open subsets of $X$ such that $X_{N}$ is a proper subset of $X_{N+1}$. (Note that we allow the possibility that $m\left(X_{1}\right)$ is infinite.) Then $\operatorname{Diff}_{\mathrm{c}}(X)=\bigcup \operatorname{Diff}_{\mathrm{c}}\left(X_{N}\right)$.

The usual orthogonal action of $\operatorname{Diff}_{\mathrm{c}}\left(X_{N}\right)$ on the real Hilbert space $L_{\mathbb{R}}^{2}\left(X_{N}\right)$, formed relative to Lebesgue measure $m$, gives rise to the standard representation $T$ of the diffeomorphism group on the complex Hilbert space $L^{2}\left(X_{N}\right)$, where

$$
[T(\psi) f](x)=J_{\psi}(x)^{1 / 2}(x) f\left(\psi^{-1} x\right), \quad f \in L^{2}\left(X_{N}\right),
$$

and $J_{\psi}(x)$ denotes the Radon-Nikodym derivative $d m\left(\psi^{-1} x\right) / d m$.

3.2. Definition. Let $Y$ be an open connected subset of $X$. We call a representation $\pi$ of $\operatorname{Diff}_{\mathrm{c}}(Y)$ tame if it is the restriction of a holomorphic tame representation of $U\left(L^{2}(Y)\right)$ to the image of $\operatorname{Diff}_{\mathrm{c}}(Y)$ under $T$.

We note that the restriction of an irreducible holomorphic tame representation of $U\left(L^{2}(Y)\right)$ to the image $T\left[\operatorname{Diff}_{\mathrm{c}}(Y)\right]$ is always irreducible. These are the representations studied by Goldin, Sharp, Menikoff, Grodnik, and Powers [8, 9, 10, 14, 15], Kirillov [13], and Vershik-Gel'fand-Graev [24] in the early 1970's. The explicit link with the unitary group was mentioned by Okomoto and Sakurai [17]. A Lie algebra approach to these representations has been developed by P. Chernoff [5].

We call a representation of $\operatorname{Diff}_{\mathrm{c}}(X)$ locally tame relative to $\mathcal{F}$ if its restriction to the subgroup $\operatorname{Diff}_{\mathrm{c}}\left(X_{N}\right)$ is always tame. Note that a locally tame representation is always locally weakly continuous (cf. [11]). 
3.3. Proposition. Let $\pi$ be a holomorphic representation of $\mathcal{U}$, where $\mathcal{U}$ is formed by the inductive limit of the unitary groups of the complex Hilbert spaces $L^{2}\left(X_{N}\right)$. Then:

(1) $\pi \circ T$ is a locally tame representation of $\operatorname{Diff}_{\mathrm{c}}(X)$;

(2) the von Neumann algebras generated by $\pi(\mathcal{U})$ and $\pi\left(T\left[\operatorname{Diff}_{\mathrm{c}}(X)\right]\right)$ coincide.

Proof. Consider the holomorphic representation $\pi$ of $\mathcal{U}$. Then the restriction $\pi \mid U\left(L^{2}\left(X_{N}\right)\right)$ is tame [12]. In particular, $\pi \circ T$ is locally tame, so (1) holds. Next, by Corollary 1.4 and [17], we know that $\pi\left(U\left(L^{2}\left(X_{N}\right)\right)\right.$ and $\pi\left(T\left[\operatorname{Diff}_{\mathrm{c}}\left(X_{N}\right)\right]\right)$ generate the same von Neumann algebras. By Lemma 1.3, statement $(2)$ follows.

3.4. Corollary. If the measure of $X_{1}$ is infinite, then the von Neumann algebras generated by $\pi(\mathcal{U})$ and $\pi\left(T\left[\operatorname{SDiff}_{\mathrm{c}}(X)\right]\right)$ coincide, where $\operatorname{SDiff}_{\mathrm{c}}(X)$ consists of all measure preserving diffeomorphisms in $\operatorname{Diff}_{\mathrm{c}}(X)$.

Proof. By [24, Theorem 1.2], we know that the restriction of a tame representation of $\operatorname{Diff}_{\mathrm{c}}\left(X_{N}\right)$ to $\operatorname{SDiff}_{\mathrm{c}}\left(X_{N}\right)$ generates the same von Neumann algebra if the measure of $X_{N}$ is infinite. The result follows from Lemma 1.3.

Comments. (1) The collection of representations depends on the choice of the orthonormal system $\left\{f_{n}\right\}$. One possible choice for this system would be a sequence of characteristic functions of disjoint sets of unit measure.

(2) The matrix $\left[T(\psi) f_{m}, f_{n}\right]_{1 \leq m, n<\infty}$ is a generalization of the intersection matrix of a partition due to A. M. Vershik.

(3) This method of constructing representations of the diffeomorphism group falls naturally in the framework described in [15].

(4) We can work with the group, denoted by $\mathcal{G}$, formed by taking the semidirect product of $\operatorname{Diff}_{\mathrm{c}}(X)$ with $\mathcal{C}_{\mathrm{c}}^{\infty}(X)$. We find that the generalized characters naturally extend to this semidirect product group. To see this, let $M(f)$ denote the representation of $\mathcal{C}_{\mathrm{c}}^{\infty}\left(X_{N}\right)$ on $L^{2}\left(X_{N}\right)$ given by $e^{i f(x)} g(x)$, where $g \in L^{2}\left(X_{N}\right)$. Then $(\psi, f) \mapsto T(\psi) M(f)$ is a representation of $\mathcal{G}$ that actually embeds this group into $\mathcal{U}$. We then find that the generalized characters introduced for $\operatorname{Diff}_{\mathrm{c}}(X)$ extend to the semidirect product as

$$
E(\psi, f)=\widehat{\chi}[\Phi(T(\psi) M(f))] .
$$

Further, we find that the corresponding cyclic representation $\pi_{E}$ of $\mathcal{G}$ generates exactly the same von Neumann algebra as $\operatorname{Diff}_{\mathrm{c}}(X)$ and $\mathcal{U}$ itself. Note that D. Pickrell [21] has explored Mackey analysis for infinite-dimensional semidirect products. We summarize these remarks:

3.5. Proposition. Let $\mathcal{G}$ be the semidirect product of $\operatorname{Diff}_{\mathrm{c}}(X)$ with $\mathcal{C}_{\mathrm{c}}^{\infty}(X)$. Then the positive-definite functions $E(\psi, f)=\widehat{\chi}[\Phi(T(\psi) M(f))]$ generate semifinite factor representations of $\mathcal{G}$. 
Discussion of the $N / V$ limit. These infinite volume limits can be naturally described in our framework. For a discussion of these limits see $[8,10,14]$. We first observe that if $L^{\prime}$ is a real Hilbert space with a non-zero vector $\omega$, then the subgroup $K^{\prime}$ of $O\left(L^{\prime}\right)$ of orthogonal transformations $W$ such that $W \omega=\omega$ can be identified with the orthogonal group of $L^{\prime} \ominus\langle\omega\rangle$. The irreducible spherical functions $\phi$ for $\left(O\left(L^{\prime}\right), K^{\prime}\right)$ are classified as

$$
\phi^{(n)}(W)=\frac{1}{\|\omega\|^{2 n}}\left(S^{n}(W) \omega^{\otimes n}, \omega^{\otimes n}\right),
$$

where $S^{n}(W)$ denotes the $n$th symmetric power of $W$. This is an old result of I. J. Schoenberg (see [20, p. 84]). (Recall that a representation $\pi$ of a group $G$ relative to a subgroup $K$ is spherical if there is a unit vector that is fixed under the action of $K$. A matrix coefficient relative to such a $K$-fixed vector is a spherical function.)

More generally, we consider an increasing sequence $L_{1} \subset L_{2} \subset \ldots$ of infinite-dimensional real Hilbert spaces whose union has completion $L$. Further, we set $\Omega_{k}=f_{1}+\ldots+f_{k}$, with $\left\|\Omega_{k}\right\|^{2}=V_{k}$. Here, $\left\{f_{j}\right\}$ is an orthogonal system (not necessarily orthonormal) such that $f_{j} \in L_{j} \ominus L_{j-1}\left(L_{0}=\{0\}\right)$.

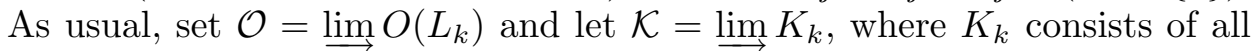
elements in $O\left(L_{k}\right)$ that fix the vector $\Omega_{k}$.

3.6. Proposition. The irreducible spherical functions of $(\mathcal{O}, \mathcal{K})$ have the form

$$
E_{\lambda}(W)=e^{\lambda([W-I] \Omega, \Omega)}, \quad \lambda \in \mathbb{R}^{+} \cup\{0\},
$$

where $\Omega$ is the formal vector $f_{1}+f_{2}+\ldots$ The function $E_{\lambda}$ is given as the limit of irreducible spherical functions of the pairs $\left(O\left(L_{k}\right), K_{k}\right)$, where $E_{\lambda}=$ $\lim _{k \rightarrow \infty} \phi_{k}^{\left(N_{k}\right)}$ if $\lambda=\lim _{k \rightarrow \infty} N_{k} /\left\|\Omega_{k}\right\|$ and where $\phi_{k}^{\left(N_{k}\right)}$ is the spherical function with index $N_{k}$ of the group $K_{k}$.

Proof. We first observe that $E_{\lambda}$ is irreducible as follows. By a result of $\left[7\right.$, p. 121] for the usual inductive limit unitary group, $E_{\lambda}$ is irreducible as a positive-definite function on $U(2 \infty)$, where $U(2 \infty)$ is given as in the statement of Lemma 2.6. By continuity, $E_{\lambda}$ extends to the group $\mathcal{U}$. Hence, it remains irreducible as a function on $\mathcal{U}$ by Lemma 2.6. Finally, by Corollary 1.4, $E_{\lambda}$ gives an irreducible representation of $\mathcal{O}$ by restriction.

We first classify the irreducible spherical functions $f$ for the pair $(G, K)$, where $G$ is an inductive limit of compact groups, since $f$ may be obtained as a limit of spherical functions of the approximating subgroups $\left(G_{k}, K_{k}^{0}\right)$ [18, Theorem 22.10]. The result will then follow since $G$ is dense in $\mathcal{O}$ and $f$ will be continuous relative to $\mathcal{O}$.

We let $G=\underline{\lim _{\longrightarrow}} O\left(L_{k}^{0}\right)$, where $L_{k}^{0} \subset L_{k} ; \operatorname{dim}\left(L_{k}^{0}\right)=2 k ; f_{1}, \ldots, f_{k} \in L_{k}^{0}$; and $\left(\bigcup L_{k}^{0}\right)^{-}=L$. Set $K_{k}^{0}=O\left(L_{k}^{0}\right) \cap K_{k}$. Let $W \in O\left(L_{k_{0}}^{0}\right)$ and assume that $\lambda=\lim _{k \rightarrow \infty} N_{k} / V_{k}$. We consider the limits of the irreducible spherical 
functions for the pairs $\left(O\left(L_{k}^{0}\right), K_{k}^{0}\right)$ :

$$
\begin{aligned}
\frac{1}{\left\|\Omega_{k}\right\|^{2}}\left(S^{N_{k}}(W) \Omega_{k}^{\otimes N_{k}}, \Omega_{k}^{\otimes N_{k}}\right) & =\frac{1}{V_{k}^{N_{k}}}\left[\left(W\left(\Omega_{k_{0}}+\Omega_{k, k_{0}}\right),\left(\Omega_{k_{0}}+\Omega_{k, k_{0}}\right)\right]^{N_{k}}\right. \\
& =\frac{1}{V_{k}^{N_{k}}}\left[\left(W \Omega_{k_{0}}, \Omega_{k_{0}}\right)+\left(V_{k}-V_{0}\right)\right]^{N_{k}} \\
& =\left[1+\frac{\left(W \Omega_{k_{0}}, \Omega_{k_{0}}\right)-V_{0}}{V_{k}}\right]^{N_{k}} \\
& \rightarrow \exp \left[\lambda\left\{\left(W \Omega_{k_{0}}, \Omega_{k_{0}}\right)-V_{0}\right\}\right] \\
& =\exp \left[\lambda\left([W-I] \Omega_{k_{0}}, \Omega_{k_{0}}\right)\right] \\
& =\exp [\lambda([W-I] \Omega, \Omega)]
\end{aligned}
$$

where $\Omega_{k, k_{0}}=\Omega_{k}-\Omega_{k_{0}}$ and $\Omega$ is the formal vector $f_{1}+f_{2}+\ldots$ Note that the inner product $([W-I] \Omega, \Omega)$ is well defined since $W f_{j}=f_{j}$ for $j$ sufficiently large. (Formally, if we let $E$ denote the algebraic inductive limit of $\bigcup L_{N}$, then we can identify $\Omega$ as giving the linear functional $x \mapsto(x \Omega, \Omega)$ on $E$.) Hence, any irreducible spherical function of $(\mathcal{O}, \mathcal{K})$ has the desired form. This ends the proof.

If we restrict the irreducible spherical functions to $\operatorname{Diff}_{c}(X)$ for representations constructed from an increasing sequence $\mathcal{F}$ of subsets $X_{1} \subset X_{2} \subset \ldots$ with $f_{j}=\chi_{X_{j}}-\chi_{X_{j-1}}\left(\right.$ take $\left.X_{0}=\emptyset\right)$, then $\Omega_{j}=\chi_{X_{j}}$. We find that

$\lim _{k \rightarrow \infty} \phi_{k}^{\left(N_{k}\right)}(T(\psi))=\exp [([T(\psi)-I] \Omega, \Omega)]=\exp \left[\int_{X}\left(\{\operatorname{det} \psi(x)\}^{1 / 2}-1\right) d x\right]$,

which is the functional for the free boson gas given in [8] and is called the Poisson function in [24]. These representations have also been systematically studied in [11] and [16].

We include the following result because of an obscurity in $[8$, p. 95, (3.31)] that any representation of $\mathcal{G}$ can be decomposed into factor representations. This is not true for any infinite-dimensional group; for example, the self-representation of the unitary group of the abelian von Neumann algebra $L^{\infty}([0,1])$, relative to Lebesgue measure, on $L^{2}([0,1])$ cannot be decomposed into a direct integral of irreducible representations which are strongly continuous.

We give a sketch of the existence of direct integral decompositions for inductive limit groups which is an adaptation of the case for separable locally compact groups, using the framework given in [22]. Let $G_{\infty}=\underline{\lim } G_{n}$, where each $G_{n}$ is a separable metric compact group such that $G_{n}$ has Haar measure zero in $G_{n+1}$. As in [22, II.1.1], we let $L=\underline{\lim } L_{(n)}$, where $L_{(n)}=\sum_{k=1}^{n} L^{1}\left(G_{k}\right) \subset M\left(G_{n}\right)$, the Banach *-algebra of Borel measures on $G_{n}$. Then $L$ becomes a separable Banach ${ }^{*}$-algebra. Further, if $\mu \in L$, 
then $\mu=\sum_{k=1}^{\infty} \mu_{k}$, where $\mu_{k} \in L^{1}\left(G_{k}\right)$ and $\|\mu\|_{L}=\sum_{k=1}^{\infty}\left\|\mu_{k}\right\|_{L^{1}\left(G_{k}\right)}$. By [22, II.1.5], there is a bijection between the continuous infinite-dimensional irreducible unitary representations $\pi$ of $G_{\infty}$ and the infinite-dimensional irreducible *-representations $\pi^{\prime}$ of $L$. In particular, for such a $\pi$, we find $\pi^{\prime}(\mu)=\int_{G_{\infty}} \pi(g) d \mu(g)$ if $\mu \in L^{1}\left(G_{k}\right)$ (here, $G_{k} \subset G_{\infty}$, so we treat $\mu$ as a measure on $\left.G_{\infty}\right)$. Write $\Phi(\pi)=\pi^{\prime}$.

We now fix a separable infinite-dimensional Hilbert space $H$. Let $\operatorname{Irr}_{\infty}\left(G_{\infty}\right)$ denote the set of all infinite-dimensional unitary representations of $G_{\infty}$ on $H ; \operatorname{Irr}_{\infty}(L)$ is defined similarly. Further, we recall that $\operatorname{Irr}_{\infty}(L)$ is a standard Borel space such that $\pi^{\prime} \mapsto\left(\pi^{\prime}(x) v, w\right)$ is a Borel function for all $x \in L$ and $v, w \in H$. As for separable locally compact groups, we give $\operatorname{Irr}_{\infty}\left(G_{\infty}\right)$ the smallest Borel structure such that $\pi \mapsto(\pi(g) v, w)$ is Borel for all $g \in G_{\infty}$ and $v, w \in H$. We claim that $\Phi: \operatorname{Irr}_{\infty}\left(G_{\infty}\right) \rightarrow \operatorname{Irr}_{\infty}(L)$ is a Borel isomorphism. Without loss of generality, we consider $\mu \in L$ of the form $\mu \in L^{1}\left(G_{k}\right)$. Then $\mu$ is the weak *-limit of the finite sums $\sum_{j=1}^{m} c_{j}^{(m)} \delta_{g_{j}^{(m)}}$. So, we have

$$
\begin{aligned}
\left(\pi^{\prime}(\mu) v, w\right) & =\left(\int_{G_{\infty}} \pi(g) d \mu(g) v, w\right)=\left(\int_{G_{k}} \pi(g) d \mu(g) v, w\right) \\
& =\lim _{m \rightarrow \infty} \sum_{j=1}^{m} c_{j}^{(m)}\left(\pi^{\prime}\left(g_{j}^{(m)}\right) v, w\right) .
\end{aligned}
$$

Hence, $\Phi$ is a Borel mapping. The approximate identites given in [22, II.1.2] can be used to show that $\Phi^{-1}$ is also Borel. We conclude that these Borel spaces are isomorphic. With these observations, the generalization of Theorem 18.7.4 in [6], which gives the equivalence between direct integral decompositions of a separable locally compact group and its group algebra, holds for $G_{\infty}$ and $L$ as well.

3.7. Proposition. (a) Let $\pi$ be a spherical representation of $(\mathcal{O}, \mathcal{K})$. Then $\pi$ is a direct integral of irreducible spherical representations; that is, $\pi$ is unitarily equivalent to $\int_{[0, \infty)}^{\oplus} \pi_{\lambda} d \mu(\lambda)$, where $\mu$ is a positive measure on $[0, \infty)$ and $\pi_{\lambda}$ is the irreducible spherical representation corresponding to $E_{\lambda}$.

(b) The restriction of an irreducible spherical representation of $\mathcal{O}$ to $\operatorname{Diff}_{\mathrm{c}}(X)$ is still irreducible.

(c) The direct integral decomposition of a spherical representation of $(\mathcal{O}, \mathcal{K})$ is preserved under restriction to the image of $\operatorname{Diff}_{\mathrm{c}}(X)$.

Proof. Let $\pi$ be a spherical representation of $(\mathcal{O}, \mathcal{K})$ with a unit cyclic vector $v$ which is fixed under the action of $\mathcal{K}$. Now the commutant of $\{\pi(\mathcal{O})\}$ is abelian by an application of Lemma 1.3 because the commutant of any spherical representation of $\left(O\left(L_{N}\right), K_{N}\right)$ is abelian. As in the proof of Propo- 
sition 3.6, we use the inductive limit groups $(G, K)$. Now we find that $v$ is still cyclic and $K$-fixed. By the above discussion, $\pi$ admits a direct integral decomposition $\int_{Z}^{\oplus} \pi(\zeta) d \mu(\zeta)$, where $\pi(\zeta)$ are irreducible representations a.e. (note: $\pi(\zeta)$ are either infinite-dimensional or the trivial representation) by $[6,8.5 .2]$. By writing the cyclic vector $v$ as $\int_{z}^{\oplus} v(\zeta) d \mu(\zeta)$, we find that the irreducible representations $\pi(\zeta)$ are spherical a.e. Hence, $\pi \simeq \int_{[0, \infty)}^{\oplus} \pi_{\lambda} d \mu(\lambda)$.

Statement (b) follows at once from Corollary 1.4, since an irreducible spherical representation is locally tame. For part (c), we apply parts (a) and (b). Note that the restrictions of inequivalent spherical representations $\pi_{\lambda}$ remain inequivalent on the image of $\operatorname{Diff}_{\mathrm{c}}(X)$.

The as ymptotic character formula as an $N / V$ limit. Recall that the asymptotic character formula $[3,24]$ implies that for a fixed finite holomorphic character $\chi$ and for a fixed $W \in U\left(V_{0}\right)$ there exists a sequence of normalized characters $\tilde{\chi}_{V}$ such that $\tilde{\chi}_{V}(W) \rightarrow \chi(W)$. In fact, such a sequence can be chosen relative to the statistics of the signature $\lambda_{V}$. Let $r_{j}\left(\lambda_{V}\right)$ denote the length of its $j$ th row; $c_{j}\left(\lambda_{V}\right)$ the length of its $j$ th column; and $\left|\lambda_{V}\right|$ the sum of the entries of the signature. Then $\tilde{\chi}_{V}$ converges if and only if

$$
\lim _{V \rightarrow \infty} \frac{r_{j}\left(\lambda_{V}\right)}{V}=\alpha_{j}, \quad \lim _{V \rightarrow \infty} \frac{c_{j}\left(\lambda_{V}\right)}{V}=\beta_{j}, \quad \lim _{V \rightarrow \infty} \frac{\left|\lambda_{V}\right|}{V}=\gamma .
$$

Further, we find that $\chi(W)=\operatorname{det}[f(W)]$, where $f \in \mathcal{M}$ has the form $f(z)=$ $e^{\gamma(z-1)} \times \prod_{j=1}^{\infty}\left(1-\alpha_{j}+\alpha_{j} z\right)\left(1+\beta_{j}-\beta_{j} z\right)$.

Since characters are central functions, we may view this limiting process as taking place over the set of eigenvalues $S^{1} \times \ldots \times S^{1}$ instead of $U\left(V_{0}\right)$. Further, we know that $\widetilde{\chi}_{V}$ is a sequence of uniformly bounded holomorphic polynomials in $V_{0}$ complex variables and $\chi$ itself is a meromorphic function in $V_{0}$ complex variables given in 2.9 .

Therefore, we also have pointwise convergence of the holomorphic extensions of the normalized characters, i.e., of $\widetilde{\chi}_{V}$ to $\chi$ over the set $D^{1} \times \ldots \times D^{1}$, where $D^{1}$ denotes the unit disk in the complex plane. In particular, we find that for fixed $W \in \Gamma\left(V_{0}\right)$, the set of contractions, $\widetilde{\chi}_{V}(W) \rightarrow \operatorname{det}[f(W)]$, where $f=f_{\chi}$ is the meromorphic function of one complex variable that defines the finite character $\chi$.

Finally, we let $\hat{\chi} \circ \Phi$ be a generalized character of $\mathcal{U}$. Consider a fixed element $W \in \mathcal{U}$, so that $W \in U\left(H_{V_{0}}\right)$. Then $\Phi(W) \in \Gamma\left(V_{0}\right)$. But we know already that there exists a sequence of normalized characters $\tilde{\chi}_{V}$ such that $\tilde{\chi}_{V}(\Phi(W)) \rightarrow \widehat{\chi}(\Phi(W))$.

For easy comparison with the $N / V$ limit, we consider the case $\chi(W)=$ $\operatorname{det}\left[f_{+}(W)\right]$ for $f_{+}(z)=1 /(1+\beta-\beta z)$. The corresponding finite factor representation is an inductive limit of symmetric algebras. Now, let $\beta=$ 
$\lim _{V \rightarrow \infty} N_{V} / V$, where $V$ and $N_{V}$ are positive integers. We set

$$
\tilde{\chi}_{V}(W)=\operatorname{Tr}\left[S^{N_{V}}\left(\Phi_{V}(W)\right)\right] / \operatorname{dim}\left(S^{N_{V}}\left(\mathbb{C}^{V}\right)\right),
$$

where $W \in U\left(L^{2}\left(X_{V}\right)\right)$; so $V$ gives the volume of $X_{V}$. Then $\tilde{\chi}_{V}(W) \rightarrow$ $\widehat{\chi}(\Phi(W))$; in particular, $\widetilde{\chi}_{V}(T(\psi)) \rightarrow \widehat{\chi}(\Phi(T(\psi)))$.

REMARK. After this paper was written, the article by N. Landsman, Representations of the infinite unitary group from constrained quantization in J. Nonlinear Math. Phys. 6 (1999), 161-180, appeared that describes how Rieffel-style induction can be used to give representations of $U(\infty)$. This procedure complements the concept of complete positivity for non-linear functionals.

\section{References}

[1] W. Arveson, Nonlinear states on $C^{*}$-algebras, in: Operator Algebras and Mathematical Physics, P. Jorgensen and P. Muhly (eds.), Contemp. Math. 62, Amer. Math. Soc., 1987, 283-343,

[2] R. P. Boyer, Infinite traces of AF-algebras and characters of $U(\infty)$, J. Operator Theory 9 (1983), 205-236.

[3] - Characters and factor representations of the infinite classical groups, ibid. 28 (1992), 281-307.

[4] - Generalized characters of $U(\infty)$, in: Algebraic Methods in Operator Theory, P. Jorgensen and R. Curto (eds.), Birkhäuser, 1994, 225-235.

[5] P. Chernoff, Irreducible representations of infinite dimensional transformation groups and Lie algebras I, J. Funct. Anal. 130 (1995), 255-282.

[6] J. Dixmier, Les $C^{*}$-algèbres et leurs représentations, Gauthier-Villars, Paris, 1964.

[7] I. M. Gel'fand and M. I. Graev, Principal representations of the group $U(\infty)$, in: Representation of Lie Groups and Related Topics, A. M. Vershik and D. P. Zhelobenko (eds.), Adv. Stud. Contemp. Math. 7, Gordon and Breach, New York, 1990, 119-153.

[8] G. A. Goldin, J. Grodnik, R. T. Powers, and D. H. Sharp, Nonrelativistic current algebra in the $N / V$ limit, J. Math. Phys. 15 (1974), 88-98.

[9] G. A. Goldin, R. Menikoff, and D. H. Sharp, Particle statistics from induced representations of a local current group, ibid. 21 (1980), 650-664.

[10] G. A. Goldin and D. H. Sharp, Diffeomorphism groups and local symmetries: some applications in quantum physics, in: Symmetries in Science III, B. Gruber and F. Iachello (eds.), Plenum, 1989, 181-205.

[11] R. S. Ismagilov, Representations of certain infinite-dimensional groups, in: Representation of Lie Groups and Related Topics, A. M. Vershik and D. P. Zhelobenko (eds.), Adv. Stud. Contemp. Math. 7, Gordon and Breach, New York, 1990, 223-243.

[12] A. A. Kirillov, Representations of the infinite unitary group, Soviet. Math. Dokl. 14 (1973), 288-290.

[13] - Unitary representations of the group of diffeomorphisms and of some of its subgroups, Selecta Math. Soviet. 1 (1981), 351-372.

[14] R. Menikoff, Generating functionals determining representations of a nonrelativistic local current algebra in the $N / V$ limit, J. Math. Phys. 15 (1974), 1394-1408. 
[15] R. Menikoff and D. H. Sharp, Approximate representations of a local current algebra, ibid. 16 (1975), 2353-2360.

[16] Yu. A. Neretin, Categories of bistochastic measures and representations of some infinite-dimensional groups, Russian Acad. Sci. Sb. Math. 75 (1993), 197-219.

[17] K. Okamoto and T. Sakurai, On a certain class of irreducible unitary representations of the infinite-dimensional rotation group II, Hiroshima Math. J. 12 (1982), 181-193.

[18] G. I. Ol'shanskiŭ, Unitary representations of infinite-dimensional pairs $(G, K)$ and the formalism of R. Howe, in: Representation of Lie Groups and Related Topics, A. M. Vershik and D. P. Zhelobenko (eds.), Adv. Stud. Contemp. Math. 7, Gordon and Breach, New York, 1990, 269-463.

[19] —, Caractères généralisés de $U(\infty)$ et fonctions intérieures, C. R. Acad. Sci. Paris Sér. I Math. 313 (1991), 9-12.

[20] - , Representations of infinite-dimensional classical groups, limits of enveloping algebras, and Yangians; On semigroups related to infinite-dimensional groups, in: Topics in Representation Theory, A. A. Kirillov (ed.), Adv. Soviet Math. 2, Amer. Math. Soc., 1991, 1-66; 67-101.

[21] D. Pickrell, Mackey analysis of infinite classical motion groups, Pacific J. Math. 150 (1991), 139-166.

[22] Ş. Strătilă and D. Voiculescu, Representations of AF-algebras and of the Group $U(\infty)$, Lecture Notes in Math. 486, Springer, 1975.

[23] - - - On a class of KMS states for the unitary group $U(\infty)$, Math. Ann. 235 (1978), 87-110.

[24] A. M. Vershik, I. M. Gel'fand, and M. I. Graev, Representations of the group of diffeomorphisms, Russian Math. Surveys 30 (1975), no. 6, 1-50.

[25] A. M. Vershik and S. V. Kerov, Characters and factor representations of the infinitedimensional unitary group, Soviet Math. Dokl. 26 (1982), 570-574.

[26] D. Voiculescu, Représentations factorielles de type $\mathrm{II}_{1}$ de $U(\infty)$, J. Math. Pures Appl. 55 (1976), 1-20.

Department of Mathematics

Drexel University

Philadelphia, PA 19104, U.S.A.

E-mail: rboyer@mcs.drexel.edu

Received June 29, 1998

Updated version January 6, 2003 